\title{
Microjoule mode-locked oscillators: issues of stability and noise
}

\author{
Vladimir L. Kalashnikov ${ }^{a}$ and Alexander A. Apolonski ${ }^{b}$ \\ ${ }^{a}$ Institut für Photonik, TU Wien, Gusshausstr. 27/387, A-1040 Vienna, Austria \\ ${ }^{b}$ Ludwig-Maximilians-Universität München, D-85748 Garching, Germany, \\ Max Planck Institute of Quantum Optics, Hans-Kopfermann-Str. 1, D-85748 Garching, \\ Germany and Institute of Automation and Electrometry, SB RAS, Novosibirsk 630090, Russia
}

\begin{abstract}
In this work, for the first time to our knowledge, stability and noise of a thin-disk mode-locked Yb:YAG oscillator operating in both negative- (NDR) and positive-dispersion (PDR) regimes have been analyzed systematically within a broad range of oscillator parameters. It is found, that the scaling of output pulse energy from $7 \mu \mathrm{J}$ up to $55 \mu \mathrm{J}$ in the NDR requires a dispersion scaling from $-0.013 \mathrm{ps}^{2}$ up to $-0.31 \mathrm{ps}^{2}$ to provide the pulse stability. Simultaneously, the energy scaling from $6 \mu \mathrm{J}$ up to $90 \mu \mathrm{J}$ in the PDR requires a moderate dispersion scaling from $0.0023 \mathrm{ps}^{2}$ up to $0.011 \mathrm{ps}^{2}$. A chirped picosecond pulse in the PDR has a broader spectrum than that of a chirp-free soliton in the NDR. As a result, a chirped picosecond pulse can be compressed down to a few of hundreds of femtoseconds. A unique property of the PDR has been found to be an extremely reduced timing jitter. The numerical results agree with the analytical theory, when spectral properties of the PDR and the negative feedback induced by spectral filtering are taken into account.
\end{abstract}

\section{INTRODUCTION}

High-power ultrafast thin-disk oscillators allow energy-scalable pico-(ps) and femtosecond (fs) pulse generation at $\mathrm{MHz}$ repetition rates ${ }^{1 / 2}$ To date, the over-10 $\mu \mathrm{J}$ fs-pulses have been obtained directly from the Yb:YAG thindisk oscillators operating in the negative dispersion regime (NDR) ${ }^{3 / 4}$ The high-energy pulse generation from a thin-disk oscillator operating in the positive dispersion regime (PDR) is achievable, as well ${ }^{[5}$ The high-energy fs-pulses nowadays allow direct experiments on light-matter interactions at the intensity levels approaching $\mathrm{PW} / \mathrm{cm}^{2}\left[{ }^{6 / 7}\right.$ In particular, high-harmonic generation at such energy levels promises developing of the table-top VUV/XUV sources, which are of interest for physics, chemistry, material science, medicine, and biology. In spite of the chirped-pulse amplification systems (CPA) $\frac{8}{8}$ the energy-scalable femtosecond oscillators are more compact, simple, and less expensive. Moreover, the $\mathrm{MHz}$ repetition rates of such oscillators (versus the $\mathrm{kHz}$ ones of CPA) reduce substantially measurement time in the pump-probe experiments as well as improve signal-to-noise ratio! 1

Energy-scalability issues a challenge of the oscillator stability. From the soliton area theorem, one may estimate the pulse energy $E$ in the NDR: $E \propto \sqrt{|\beta| P_{0} / \gamma}\left[\beta\right.$ is the group-delay dispersion (GDD) coefficient, $P_{0}$ is the pulse peak power, $\gamma$ is the self-phase modulation (SPM) coefficient]. If one has to confine $P_{0}$ below some level providing the pulse stability, the pulse energy in the NDR scales as $E \propto \sqrt{\beta}$. The similar estimation for the law of energy scaling follows from the energy rate equations for a mode-locked oscillator. ${ }^{9}$ As a result, the necessary GDD value scales as square of $E$. In the PDR, the scaling law can be expressed approximately as $E \propto \beta^{2} ! 10$ The last expression suggests, that the scaling properties of the PDR excel those in the NDR. However, the detailed study of stability needs a numerical approach. For instance, a departure from the area theorem appears in the $\mathrm{NDR}^{11}$ and the mechanism of pulse destabilization in the PDR can differ from the CW-excitation considered in Refs. 10

An additional issue appears from the requirement of the pulse period stability imposed by such applications of the oscillators under consideration as pump-probe experiments, parametric mixing of the pulses from different oscillators, coherent pulse enhancement in a resonant cavity, etc. The well-known analytical theory of noises of

E-mail: kalashnikov@tuwien.ac.at, Telephone: +43-1-58801-38743 


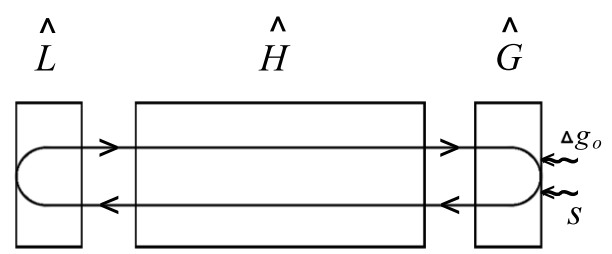

Figure 1. Block diagram for the numerical analysis of a Yb:YAG thin-disk oscillator. $\hat{L}$ is the absorption (both saturable and unsaturable), $\hat{H}$ is the compensating GDD and the SPM in air, $\hat{G}$ is the frequency-dependent saturable gain with fluctuating gain coefficient, quantum noise, GDD, and SPM.

mode-locked oscillators ${ }^{13}$ is based on the soliton perturbation theory and, thereby, does not deal with the highenergy regimes, where high-order dissipative nonlinearities contribute, and with the PDR, where there exists no chirp-free pulse. Moreover, large gain coefficient and narrow gain bandwidth distinguish the thin-disk oscillators. As a result, the gain fluctuations affect the timing jitter deeply!14 These effects, which are beyond the scope of the solitonic model, need a numerical consideration! 14

In this work, the stability thresholds and fluctuations of pulse group-delay (timing jitter) in a high-energy Yb:YAG thin-disk oscillator operating in both NDR and PDR are analyzed systematically on basis of numerical simulations. Two noise sources are considered: gain fluctuations and quantum noise due to spontaneous emission in an active medium. It is found, that the scaling properties of the PDR exceed those of the NDR in the sense, that i) the GDD value providing the pulse stability is substantially reduced in the PDR and ii) scales as $\propto \sqrt{E}$ vs. $\propto E$ in the NDR. The pulse duration is smaller in the NDR, but the spectrum is broader in the PDR that makes the chirped pulse to be compressible down to sub-picosecond pulse duration. The group delay caused by the gain dispersion is sensitive to the gain fluctuations that results in the timing jitter. However, the timing jitter is substantially reduced in the PDR as compared to that in the NDR. But in contrast to the latter, the timing jitter increases with the energy $E$ for the chirped pulse.

\section{MODEL OF MODE-LOCKED OSCILLATOR WITH NOISE}

Evolution of time- $(t)$ dependent slowly-varying field envelope $a(t)$ inside an oscillator is modeled on basis of the undistributed map shown in Fig. 1. Here the nonlinear operator

$$
\hat{L}=\exp \left[-\ell-\frac{\kappa}{1+\zeta|a(t)|^{2}}\right]
$$

describes the net-loss action. The unsaturable loss coefficient $\ell=0.07$ includes the output loss $(=12 \%)$. The nonlinear (saturable) part of the loss operator describes an action of a semiconductor saturable absorber with the modulation depth coefficient $\kappa=0.005$ and the inverse saturation power $\zeta=T_{r}^{a} / E_{s}^{a} S_{a} \approx 0.71 \mathrm{MW}^{-1}$, where the absorber relaxation time $T_{r}^{a}$ equals to $0.5 \mathrm{ps}$, the absorption saturation fluency $E_{s}^{a}$ equals to $90 \mu \mathrm{J} / \mathrm{cm}^{2}$, and the mode area $S_{a}$ corresponds to the $1 \mathrm{~mm}$ mode size on an absorber.

The Hamiltonian operator

$$
\hat{H}=i \beta \frac{\partial^{2}}{\partial t^{2}}-i \gamma_{a i r}|a(t)|^{2}
$$

describes the distributed action of the air nonlinearity and the GDD of the cavity. The GDD coefficient $\beta$ is variable $(\beta<0$ corresponds to the NDR, $\beta>0$ corresponds to the PDR). The SPM in air is defined by the coefficient $\gamma_{\text {air }}=3 \mathrm{GW}^{-1}$, that corresponds to the effective mode size of $2.2 \mathrm{~mm}$ and the $11 \mathrm{MHz}$ oscillator repetition rate.

The integro-differential stochastic operator $\hat{G}$ means 


$$
\hat{G}[a]=i \beta_{g} \frac{\partial^{2}}{\partial t^{2}} a(t)-i \gamma_{g}|a(t)|^{2} a(t)+\left(\frac{\left(g_{0}+\Delta g_{0}\right) \Omega_{g}}{1+2 \frac{\int_{-\infty}^{\infty}|a(t)|^{2} d t}{E_{s}^{g}}} \int_{t}^{\infty} \exp \left[-\Omega_{g}\left(t^{\prime}-t\right)\right] a\left(t^{\prime}\right) d t^{\prime}\right)+s(t)
$$

and describes a $200 \mu \mathrm{m} \mathrm{Yb:YAG} \mathrm{thin-disk} \mathrm{with} \mathrm{the} \mathrm{GDD} \mathrm{coefficient} \beta_{g}=260 \mathrm{fs}^{2}$ and the SPM coefficient $\gamma_{g}=0.12$ $\mathrm{GW}^{-1}$ for the mode size of $2.4 \mathrm{~mm}$. The saturable gain with the gain coefficient $g_{0}$ for a small signal and the saturation energy $E_{s}^{g}=\frac{h \nu}{\sigma T_{r}^{g}} T_{c a v} S_{g} \approx 0.24 \mathrm{~mJ}\left(T_{r}^{g}=1 \mathrm{~ms}\right.$ is the gain relaxation time, $\sigma=2 \times 10^{-20} \mathrm{~cm}^{2}$ is the gain cross-section, $T_{c a v}$ and $S_{g}$ are the cavity period and the mode area, respectively) has the causal Lorentz spectral profile $\mathrm{e}^{179}$ with the width $\Omega_{g}=5.3 \mathrm{THz}$ (the gain bandwidth of $6 \mathrm{~nm}$ ).

The stochastic (white-noise) value $\Delta g_{0}$ describes the initial gain fluctuation (in the limits of $\pm 0.025 g_{0}$ ) so that the value $g_{0}+\Delta g_{0}$ differs for each independent simulation with 10000 cavity round-trips and some fixed set of the parameters $\left(g_{0}, \beta, E_{s}^{g}\right.$, etc.). The complex stochastic value $s(t)$ such that ${ }^{14}$

$$
\left\langle s(t) s^{*}\left(t^{\prime}\right)\right\rangle=2(\ell+\kappa) \theta \frac{h \nu}{\delta t} \delta\left(t-t^{\prime}\right)
$$

describes the quantum noise of an active medium ( $\theta$ is the enhancement factor due to an incomplete inversion of active medium, $\delta t$ is the time step in subdividing of time window representing $a(t)$ ). by

The propagation of the field with the complex envelope $a(t)$ through the system shown in Fig. 1 is described

$$
a_{k+1}(t)=\frac{\hat{L}}{2} \frac{\hat{H}}{2} \hat{G} \frac{\hat{H}}{2} \frac{\hat{L}}{2} a_{k}(t)
$$

where $k$ is the cavity round-trip number. The iterative Eq. (5) is solved on basis of the symmetrized split-step Fourier method on the mesh with the minimum time step $\delta t=2.5$ fs and the simulation window of $\approx 655$ ps. The steady-state solution reached after 10000 round-trips is considered as the initial condition for the statistics gathering from the 64 independent samples of propagations with 10000 round-trips for each set of parameters of Eq. (5). The energy scaling can be provided by scaling of i) mode-size $S_{g}$, ii) cavity period $T_{\text {cav }}$, and iii) average power $P_{a v}$ that affects both $g_{0}=(\ell+\kappa)\left(1+2 P_{a v} T_{c a v} / E_{s}^{g}\right)$ and $E_{s}^{g}$.

\section{RESULTS AND DISCUSSION}

In this section, the stability and the statistic properties of a mode-locked Yb:YAG thin-disk oscillator will be analyzed in both NDR and PDR. A circulating pulse will be treated as a dissipative soliton (DS) of Eq. (5). This equation can be considered as the undistributed generalization of the nonlinear complex Ginzburg-Landau equation, which is the master equation for modeling of mode-locked solid-state and fiber oscillators. 20.21$]$

\subsection{Stability threshold in NDR and PDR}

The dependence of the GDD coefficient $\beta$ providing the DS stability on the output energy $E$ is shown in Fig. 2 for the NDR (gray curve) and the PDR (black curve). The DS is stable above the black curve for the PDR and below the gray curve for the NDR.

For the NDR, the numerical threshold GDD (gray curve in Fig. 2) scales approximately as $\beta\left[\mathrm{ps}^{2}\right] \approx$ $-0.0036(E[\mu \mathrm{J}])^{1.7}$, that is slower than the simple square law suggests (see Sec. 10. For the PDR, the numerical threshold GDD (black curve in Fig. 2) scales approximately as $\beta\left[\mathrm{ps}^{2}\right] \approx 0.0011(E[\mu \mathrm{J}])^{0.5}$, that is it obeys the square root law (see Sec. 1). The analytical distributed model of Refs $\frac{10] 22}{12}$ predicts that the threshold intracavity energy $E_{t h}^{i n}$ in the PDR obeys

$$
E_{t h}^{i n}=\frac{3 \gamma}{\kappa \zeta^{2} c^{2} \Omega_{g} \sqrt{\kappa}}\left[\sqrt{\frac{6(2-c)}{c}}-\frac{3(2+c(c-2))}{\sqrt{1-c(c-3)}} \operatorname{arctanh}\left(\frac{\sqrt{6 c(2-c)}}{2 \sqrt{1-c(c-3)}}\right)\right],
$$




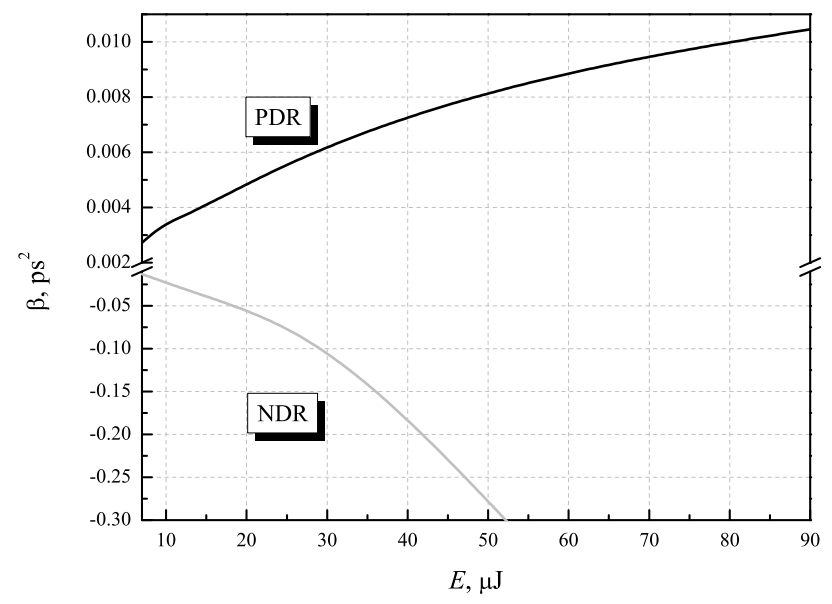

Figure 2. Threshold GDD coefficients $\beta$ providing the stable DS for the NDR (gray curve) and the PDR (black curve) in dependence on the output energy $E$. Stable pulses exist above the black curve for the PDR and below the gray curve for the NDR.
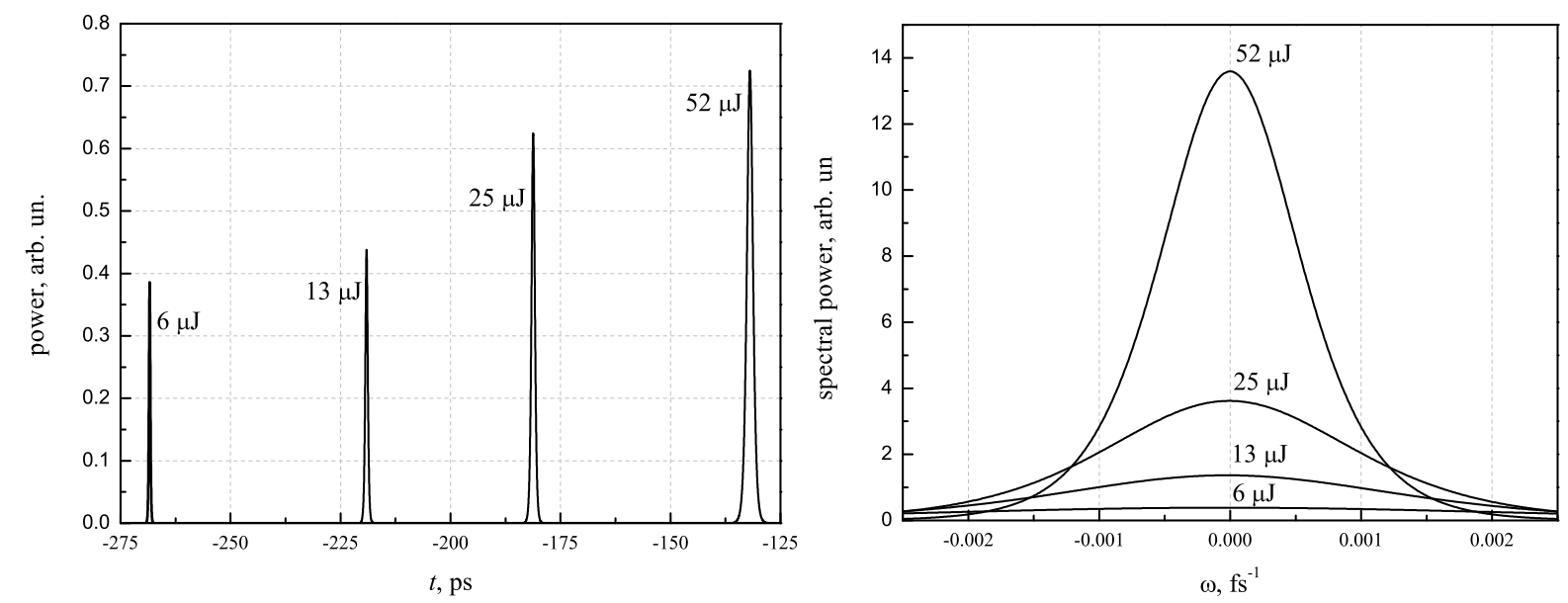

Figure 3. Instantaneous power $|a(t)|^{2}$ (left) and spectral power (right) profiles corresponding the CDs at the NDR stability border (Fig. 2). The corresponding energies are superscribed. The frequency $\omega$ corresponds to deviation from the gain maximum.

where $c=\alpha \gamma / \beta \kappa \zeta<2$. However in the case under consideration (relatively low $\kappa \zeta$ and large $\gamma$ ), the last condition is satisfied starting from the energy $E>120 \mu \mathrm{J}$ and the GDD $\beta>0.015 \mathrm{ps}^{2}$.

The destabilization scenario for both NDR and PDR is multiple pulse generation. For the PDR, the Q-switch mode-locking instability appears, as well. As one may see from Fig. 2. the GDD providing the pulse stabilization is substantially lower in the PDR than that in the NDR and such a difference increases with $E$. That is the chirped DS is more robust within a whole range of energy than the chirp-free (Schrödinger) soliton. It should be

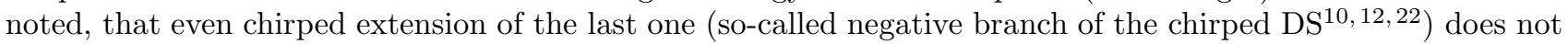
provide an effective energy scaling.

Fig. 3 shows some power and spectral power profiles corresponding to the DSs at the stability border of the NDR (gray curve in Fig. 21). One can see, that the pulse duration increases with energy in agreement with the area theorem: $T \propto|\beta| / E \propto \sqrt{|\beta|}$ (for a more careful inspection see next subsection). Simultaneously, the source of deviation from the square law $E \propto \beta^{2}$ is clearly visible: the peak power increases slightly with energy, as well (Fig. 3, left). In concordance with the pulse duration growth, the spectral width decreases with the energy increase (Fig. 3). There exists no spectral disturbance induced by the dispersion of gain medium (Eq. (3)). 

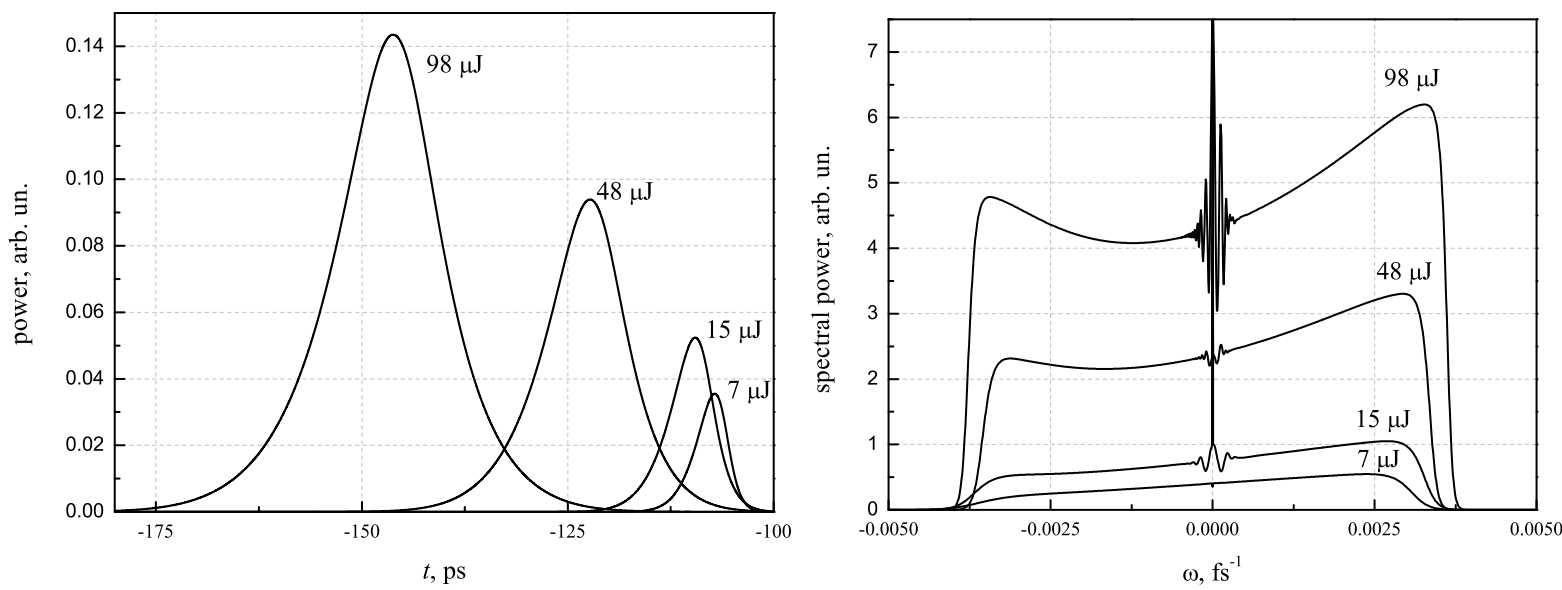

Figure 4. Instantaneous power $|a(t)|^{2}$ (left) and spectral power (right) profiles corresponding the chirped CDs at the PDR stability border (Fig. 2). The corresponding energies are superscribed. The frequency $\omega$ corresponds to deviation from the gain maximum.

The analogous profiles for the PDR are shown in Fig. 4. The DS width, the peak power, and the spectral width $\Delta$ grow with the energy in the PDR. The analytical model of Refs $\frac{102}{22}$ predicts the following relation for the last two parameters: $\gamma P_{0}=\beta \Delta^{2}$, and the $\Delta$-growth with $\beta$ corresponds to the so-called positive branch of the chirped DS, which exists along the stability threshold. The analytical theory $10[2] 22]$ predicts also that $\Delta$ increases with $\beta$ up to some maximum value of GDD and then the spectrum shortens. But such a shortening is not possible for the parametrical range under consideration. It should be noted, that $P_{0}$ is reduced and $\Delta$ is enlarged in the PDR in comparison with those parameters in the NDR.

One may see, that the spectra are asymmetrical in the PDR. Such an asymmetry has been observed experimentally in the low energy limit in Ref., 23 and we interpret it as a manifestation of the gain dispersion. ${ }^{24}$ Also, some CW-like perturbation is visible in the vicinity of spectrum center (Fig. 4). This perturbation appears in the vicinity of stability border $\underset{24}{24}$ enhances with $E$, and can be suppressed at higher GDD. The spectra are

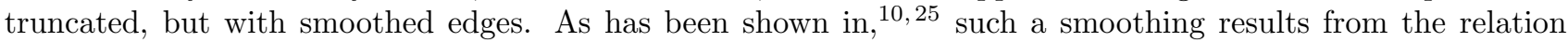
$1 / \Omega_{g}^{2}>\beta$ and $\kappa \zeta \approx \gamma$. The spectrum becomes concave with sharp edges, when the $E$ (and, correspondingly, $\beta$ ) increases.

Figs. 3 4 demonstrate the relative pulse time-delay, which value changes with $E$ but in opposite directions for the NDR and the PDR. The group-delay of a DS and its statistical properties will be considered in the next subsection.

\subsection{Group-delay of a dissipative soliton and its statistical properties}

The above described increase of $T$ with $E$ is illustrated by Fig. [5. The durations in the PDR excess those in the NDR and scale approximately as $T[\mathrm{ps}] \approx 1.5(E[\mu \mathrm{J}])^{0.51}$ (squares and black curve in Fig. 50). In the NDR, the pulse duration (circles and gray curve in Fig. 5 ) scales almost linearly with $E$ (i.e., $T[\mathrm{ps}] \approx 0.36+0.022 E[\mu \mathrm{J}]$ ) in agreement with the area theorem. However, more careful inspection shows an appearance of higher-order corrections, which enhance the pulse broadening with $E$ (the best fit is $T[\mathrm{ps}] \approx 2.4 \times 10^{-4}(90+E[\mu \mathrm{J}])^{2.7}$ ).

The group delays $\delta$ are shown in Fig. [5, as well. In the absence of gain dispersion, the group delay equals to zero by definition of the local time $t$. The gain dispersion induces a positive delay for both NDR and PDR. However, there is some additive to this delay in the PDR for shorter pulses (squares and black curve in Fig. (5)). Inversely, the group delay decreases in the NDR and such a decrease enhances for shorter pulses (circles and gray curve in Fig. 5). The energy derivatives of $\delta$ are $d \delta / d E \approx 0.5 / E^{0.86}$ and $d \delta / d E \approx-0.16 / E$ (in fs/ $\mu \mathrm{J}$ per round-trip) for the NDR and the PDR, respectively. The derivative is substantially larger for the NDR therefore one may expect that the chirped DS is more stable against the timing jitter. However, such an expectation requires a verification because the energy $E$ is not independent variable. 

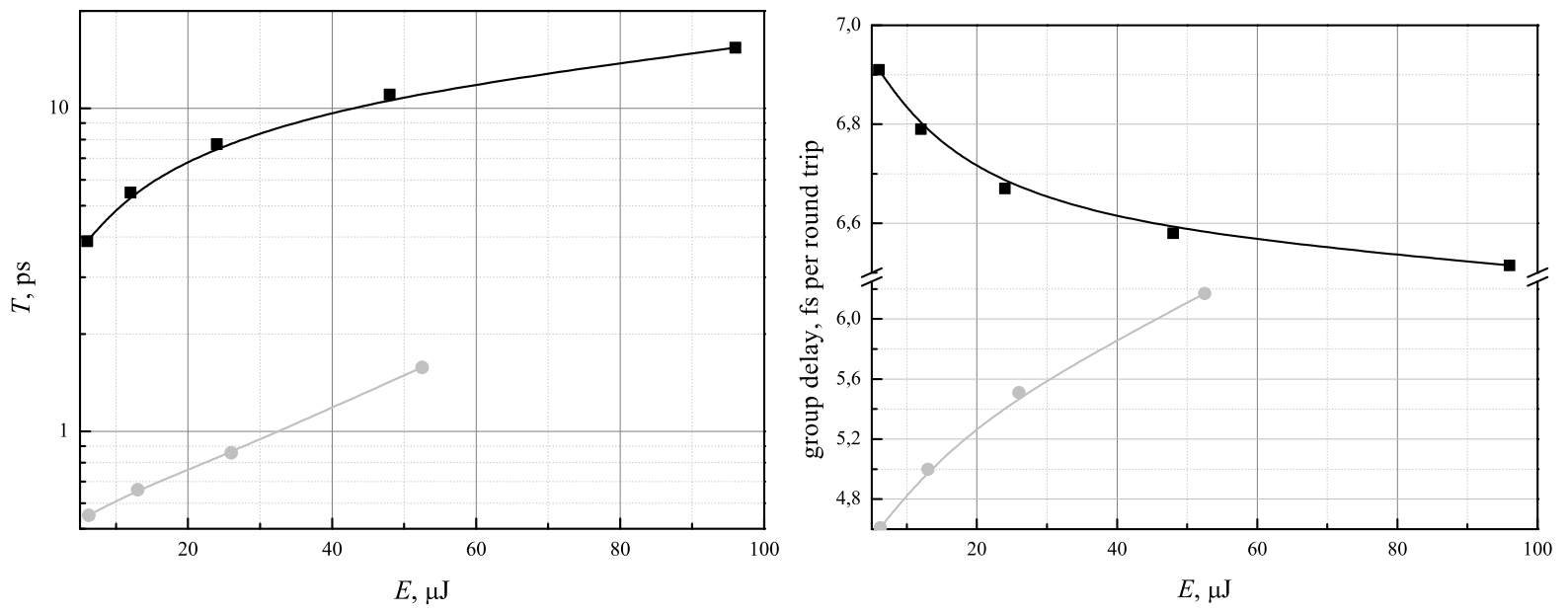

Figure 5. Pulse duration $T$ (left) and pulse group delay (right) in dependence on the output energy $E$ for the PDR (black squares and lines) and the NDR (gray circles and lines) at the stability border.

The net-variation of $\delta$ can be expressed as

$$
\Delta \delta \approx \Delta g_{0}\left[\frac{\partial \delta}{\partial g_{0}}+\frac{\partial E}{\partial g_{0}} \frac{\partial \delta}{\partial E}\right]
$$

if a source of jitter is assumed to be the gain variations $\Delta g_{0}$. In Eq. (7), we divide the total variation of $\delta$ into two parts: i) variation due to change of an amplitude of the gain dispersion, and ii) variation due to change of the pulse characteristics. The last is most interesting because it is a dynamical, i.e. nonlinear, effect. Since the "slope efficiency" $\partial E / \partial g_{0} \approx 10 E[\mu \mathrm{J}]$ for both regimes under consideration, the $\delta$-variation has to be reduced in the PDR: $\left(\partial E / \partial g_{0}\right)(\partial \delta / \partial E) \approx-1.6$ fs per round-trip in the PDR versus $\left(\partial E / \partial g_{0}\right)(\partial \delta / \partial E) \approx 5(E[\mu \mathrm{J}])^{0.14} \mathrm{fs}$ per round-trip in the NDR.

The experimental results concerning the timing jitter are discrepant $16 \mid 26]$ On the one hand, the relative intensity noise level is reduced for the PDR. On the other hand, such a reduction in Ref. ${ }^{26}$ can result from lower loss at the grating compressor in the PDR. Therefore, a further analysis of the noise properties of both PDR and NDR is required.

Our numerical modeling is based on the model of Sec. 2 with the following noise source included: the gain fluctuation and the spontaneous emission in an active medium. The first conclusion obtained from the simulations is that the contribution of quantum noise is negligible under conditions considered. As the characteristic quantity, the standard deviation of the pulse position is chosen: $\sigma(k) \equiv \sqrt{\sum_{i=1}^{N}\left(\max \left(\left|a_{k}(t)\right|^{2}\right)-\left\langle\max \left(\left|a_{k}(t)\right|^{2}\right)\right\rangle\right)^{2} / N}$, where $N=64$ is the number of statistically independent samples of the steady-state pulse propagations during $k$ cavity round-trips, $\max \left(\left|a_{k}(t)\right|^{2}\right)$ is the position of the pulse power maximum, and $\langle\ldots\rangle$ is its mean value. The simulations demonstrate a linear dependence of $\sigma(k)$ and $\left\langle\max \left(\left|a_{k}(t)\right|^{2}\right)\right\rangle$ on $k$.

The dependencies of the group delay standard deviation $\sigma$ and the DS width $T$ on the dispersion GDD are shown in Fig. 6. One can see, that the $\sigma$-parameter characterizing the timing jitter is substantially reduced in the PDR. The dependencies obtained suggest that the source of such reduction is the negative passive feedback induced by a spectral dissipation:

1) The chirped DS has a broader spectrum with a concentration of spectral energy at the spectrum edges, where dissipation is maximum (Fig. 4). The energy growth broadens the spectrum and enhances the spectral loss and vice-versa. Thus, the negative feedback works: energy growth enhances the spectral loss, energy reduction reduces the spectral loss. As a result, $\sigma$ diminishes (Fig. 6). 

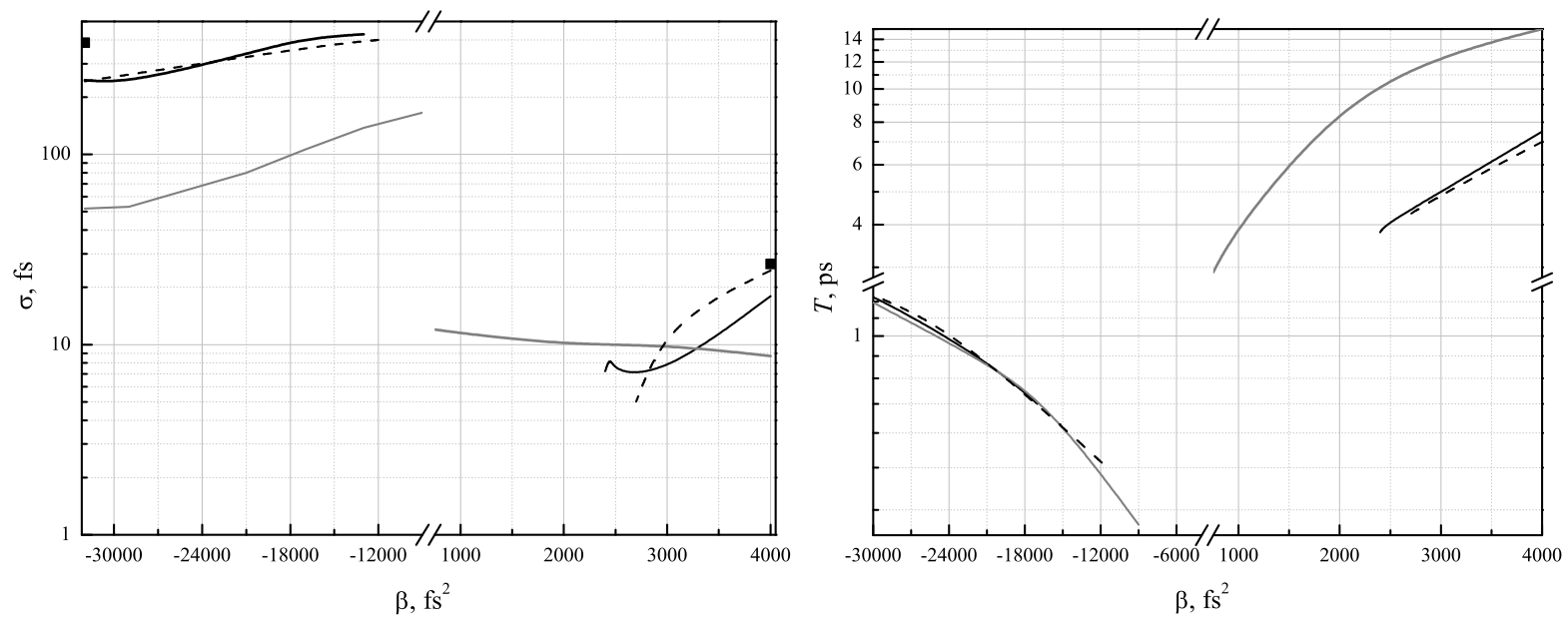

Figure 6. Jitter parameter $\sigma(k)$ (left) and $T$ (right) corresponding to the stable DS. $k=10000, \Omega_{g}=5.3$ (solid and dashed black curves as well as squares) and $10.5 \mathrm{THz}$ (gray curves). The output energy $E=6$ (dashed curves), 7 (solid curves) and $14 \mu \mathrm{J}$ (squares); $\zeta=0.71$ (solid curves and squares) and $0.35 \mathrm{MW}^{-1}$ (dashed curves). The other parameters are given in Sec. 2 ,

2) The GDD growth shortens the spectrum and the spectral loss decreases. As a result, $\sigma$ increases, that is the negative feedback disappears (the $\sigma$-value in the PDR tends to that in the NDR, black curves in Fig. 6).

3) The decrease of spectral dissipation due to gain band broadening (i.e. the $\Omega_{g}$-growth; gray curve in Fig. 6) approaches the PDR's $\sigma$ to that in the NDR. The character of dependence on $\beta$ changes, as well: $\sigma$ decreases with the $T$-growth. The decrease of $\sigma$ with the $\Omega_{g}$-growth in the NDR means that the main source of the groupdelay, that is the gain dispersion, weakens with the gainband broadening. Since the gain band of a Yb-fiber oscillator is broader than that for a Yb:YAG solid-state thin-disk oscillator, this conclusion means a reduction of difference between the noise properties of the PDR and the NDR for a fiber oscillator. ${ }^{26}$ Simultaneously, the $\sigma$-decrease with the pulse duration (or $|\beta|$ ) growth in the NDR (Fig. 6) can be explained as a result of diminishing action of a gain dispersion on the broadened chirp-free DS (such an action can be approximately described as $\left.\propto\left(1 / \Omega_{g}\right) d / d t \propto 1 / \Omega_{g} T\right)$.

The decrease of the inverse saturation power $\zeta$ does not affect the stability noticeably (dashed curves in Fig. 6). The gainband broadening $\left(\Omega_{g}\right.$-growth) enhances the stability against CW-excitation and multipulsing in the PDR (gray curve in Fig. 6) because the stability parameter is $\left.\propto \beta \Omega_{g}^{2}, 10\right] 12$ that is the stabilizing GDD is inversely proportional to $\Omega_{g}^{2}$.

The energy growth (squares in Fig. 6] correspond to the minimum $|\beta|$ providing the DS stability) increases the stabilizing GDD (see Fig. 2). The $\sigma$ variation with $E$ is different for the PDR and the NDR (Fig. 7). The standard deviation of the pulse maximum location decreases with $E$ for the NDR (the measurement in a $6 \mu \mathrm{J}$ Yb:YAG oscillator demonstrates the jitter of $125 \mathrm{fs}(29)$. One may assume that such a decrease results from the conjecture $\partial \delta / \partial g_{0} \propto 1 / T$ in Eq. (7). It should be repeated, that the noise source considered has not a quantum nature (versus Ref. $\frac{16}{16}$ for instance).

The standard deviation $\sigma$ (i.e. the jitter parameter) is substantially reduced in the PDR (Fig. 7) and equals to few femtoseconds in the vicinity of $E \approx 7 \mu \mathrm{J}$. We attribute this reduction to the negative passive feedback caused by the spectral loss (see above). In the PDR, $\sigma$ increases with $E$ despite the fact that the pulse duration increases, as well (Fig. 57). Such an effect is not incorporated in Eq. (7) and one can assume, that the source of jitter is connected with a spectrum broadening and an enhancement of spectral components located at the spectrum edges (the spectrum becomes more concave for higher energy, Fig. (4). Hence, the higher-order dispersion induced by a Lorentz gainband (which contribution in the spectral domain is $\propto 1 /\left(1-i \omega / \Omega_{g}\right)$, Eq. (3) ) affects the DS group delay. This effect will be analyzed elsewhere.

The timing jitter produced by the gain fluctuations acts destructively on the DS coherence. Figs. 8 and 9 show the averaged power and spectral power profiles as well as the corresponding coherence ratios for the 64 


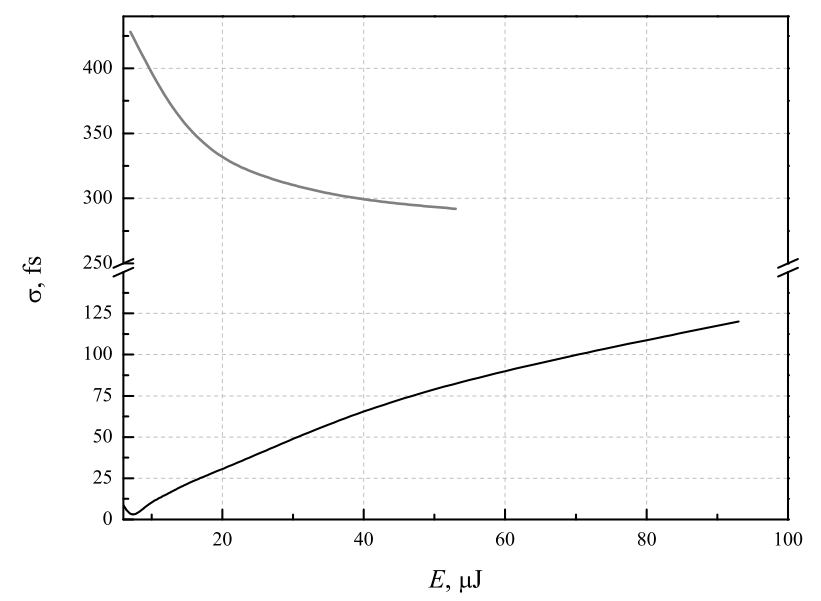

Figure 7. Standard deviation $\sigma(k)$ at the stability border (Fig. 2) in dependence on $E$ for the PDR (black curve) and the NDR (gray curve). $k=10000$.
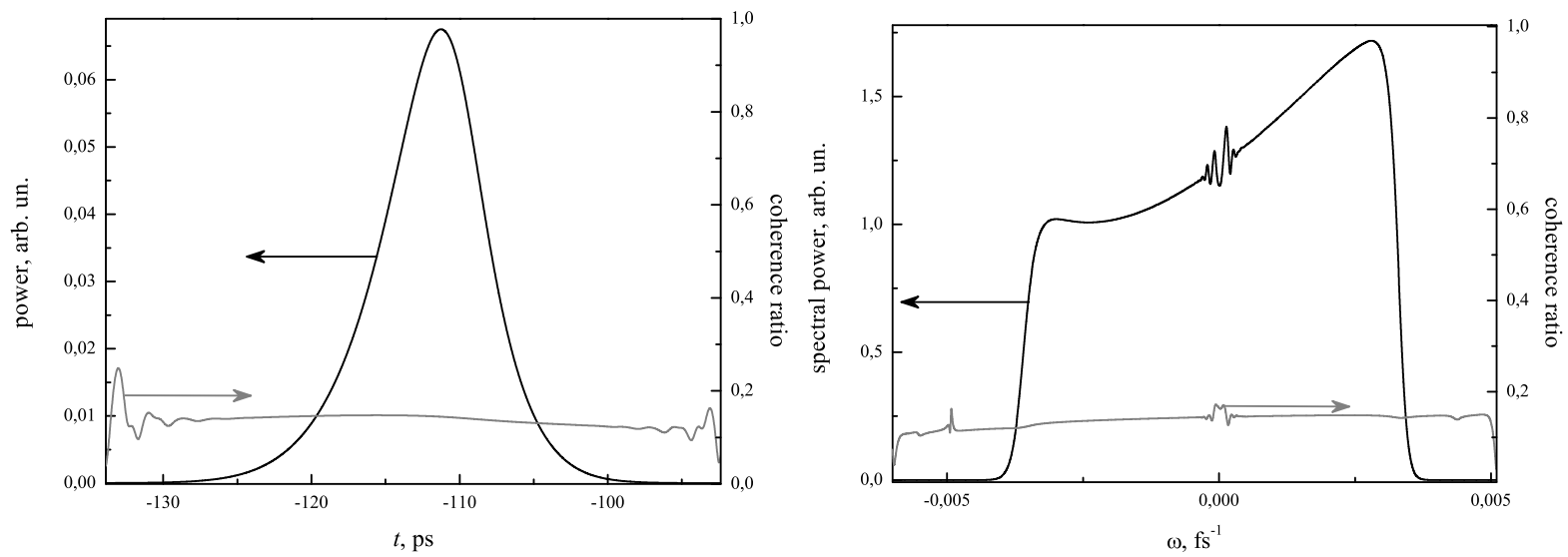

Figure 8. Averaged power (left) and spectral power (right) profiles (black curves) as well as temporal (left) and spectral (right) coherence ratios (gray curves) for the PDR at the stability border. $E=46 \mu \mathrm{J}, k=10000$.

independent propagation samples after $k=10000$ cavity round-trips. As the definition of the coherence ratio, we $u^{28}$

$$
\Gamma \equiv \frac{\left|\left\langle a_{i}^{*} a_{j}\right\rangle_{i \neq j}\right|}{\sqrt{\left\langle\left|a_{i}\right|^{2}\right\rangle_{i}\left\langle\left|a_{j}\right|^{2}\right\rangle_{j}}}
$$

where 64 independent propagation samples after 10000 cavity round-trips are deviled into $(i, j)$-pairs $(i$ and $j$ range from 1 to 32$)$. 〈... means averaging over the corresponding index. $a$ is the complex amplitude in the time or frequency domains.

One can see, that the coherence ratio (both temporal and spectral) is substantially below 1 for high-energy pulses. The NDR provides a higher coherence at the average than that in the PDR. A possible explanation is that the DS in the PDR is chirped and the power fluctuations distort the pulse phase that reduces the coherence. Simultaneously, the coherence ratio is almost constant inside a chirped DS in both time and frequency domains.

\subsection{Analytical estimations}

Complicate dynamics of the high-energy oscillators needs the cumbersome numerical simulations. Especially, gain saturation, gain dispersion, SPM and higher-order dispersions entangle the contributions from different 

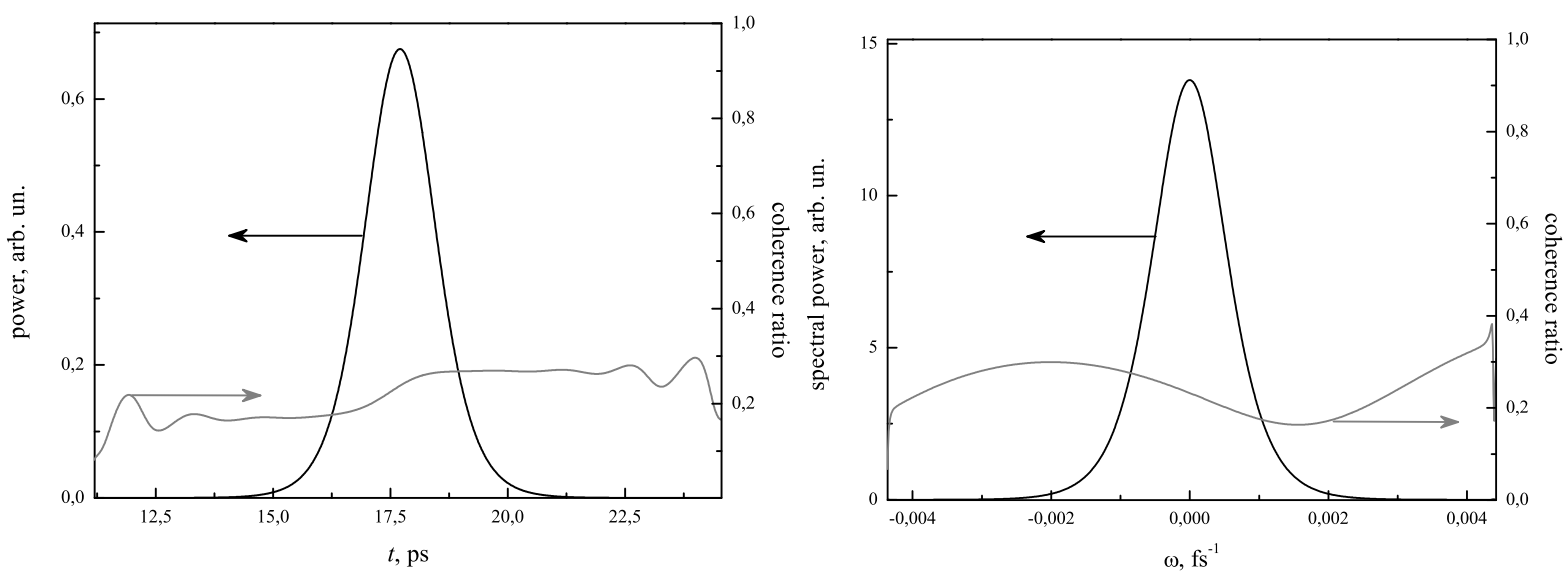

Figure 9. Averaged power (left) and spectral power (right) profiles (black curves) as well as temporal (left) and spectral (right) coherence ratios (gray curves) for the NDR at the stability border. $E=53 \mu \mathrm{J}, k=10000$.

noise sources and add the correlations between them. As will be shown below, the simple improvement of theory developed in Ref. ${ }^{14}$ allows comparison between the quantum noise limit and the timing jitter caused by gain dispersion for both PDR and NDR.

The intracavity power density of the timing noise induced by a spontaneous emission and a dispersionmediated effect of frequency fluctuations is $13[14$

$$
S(f)=\left(\frac{2 \beta}{f T_{\text {cav }}}\right)^{2} \frac{4(\ell+\kappa)}{(2 \pi f)^{2}+\tau_{g}^{-2}} \frac{h \nu}{E^{2} T_{\text {cav }}} \int \omega^{2}|\tilde{a}(\omega)|^{2} d \omega+\frac{4(\ell+\kappa)}{(2 \pi f)^{2}} \frac{h \nu}{E^{2} T_{\text {cav }}} \int t^{2}|a(t)|^{2} d t,
$$

where $f$ is the noise frequency and $\tilde{a}(\omega)$ is the Fourier image of the field. It is assumed, that the saturated gain coefficient equals to the net-loss coefficient, $E$ is the intracavity energy. The impact of a gainband as a spectral filter is defined by the parameter $\tau_{g}=\frac{T_{c a v} \Omega_{g}^{2} / \Delta^{2}}{16(\ell+\kappa)}(\Delta$ is the pulse spectral width for the truncated spectrum in the PDR or $1.763 / \sqrt{3} T$ for the NDR). Integral $\int \omega^{2}|\tilde{a}(\omega)|^{2} d \omega / E \approx \Delta^{2}$ for the PDR or $\approx 0.265(0.315 / T)^{2}$ for the NDR. Integral $2 \int t^{2}|a(t)|^{2} d t / E \approx 0.529 T^{2}$ for both regimes.

Left Fig. 10 shows the noise spectrum in the quantum limit of (9) for the PDR (black curve) and the NDR (gray curve). One can see, that the NDR has substantially higher noise, especially in the low-frequency range. Analysis of Eq. (9) demonstrates that such an excess results due to the strong noise suppression of frequency fluctuations in the PDR (first term in Eq. (9)). This results from i) more effective spectral filtering for the broad truncated spectrum of the chirped DS and ii) lower $|\beta|$ in the PDR. On the other hand, the timing effect of spontaneous emission is stronger in the PDR due to larger $T$ (second term in Eq. (99)). As a result, the difference between noise levels decreases with $f$.

As it has been shown in the previous subsection, the gain dispersion translates the gain fluctuations into the timing noise. The trivial correction of the model presented in Ref ${ }^{[14}$ with taking into account the negative feed-back induced by spectral filtering results in

$$
S(f)=\frac{1}{(2 \pi f)^{2}+\tau_{g}^{-2}}\left(\frac{1}{T_{c a v} \Omega_{g}}\right)^{2} S_{g}(f),
$$

where $S_{g}$ is the gain noise. Let's $S_{g}$ results from quasi-harmonic gain oscillations with the amplitude $0.05(\ell+\kappa)$, the frequency $\Omega$ and the Lorentz bandwidth $\Theta$. Then

$$
S_{g}(f)=[0.05(\ell+\kappa)]^{2} \sqrt{\frac{2}{\pi}} \frac{\Theta\left[\Theta^{2}+(2 \pi f)^{2}+\Omega^{2}\right]}{(2 \pi f)^{4}+\left(\Theta^{2}+\Omega^{2}\right)^{2}+2(2 \pi f)^{2}\left(\Theta^{2}-\Omega^{2}\right)}
$$



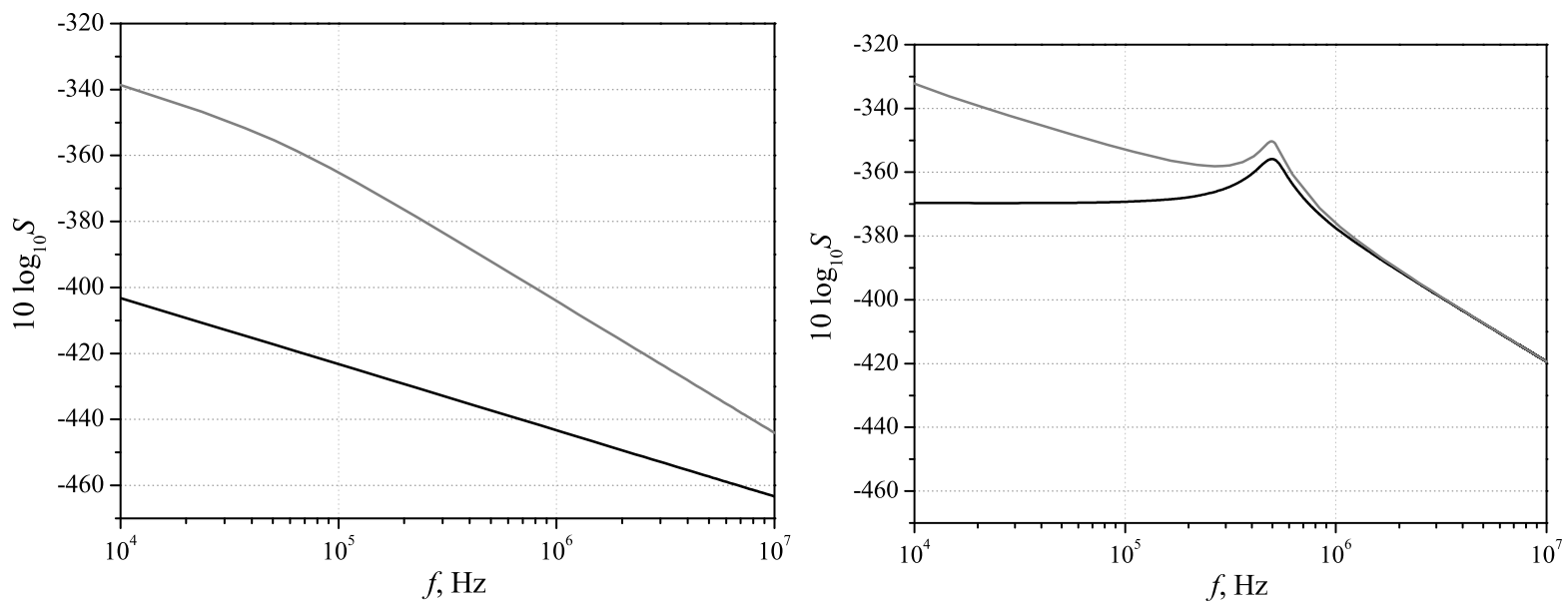

Figure 10. Quantum limit (left) for the timing noise. The NDR (gray curve) corresponds to $T=1 \mathrm{ps}, \beta=-0.01 \mathrm{ps}^{2}$, the PDR (black curve) corresponds to $\Delta=3 \mathrm{ps}^{-1}, T=3 \mathrm{ps}, \beta=0.0025 \mathrm{ps}^{2} . E=50 \mu \mathrm{J}, \Omega_{g}=5.3$ THz. Timing noise induced by gain fluctuation (right) in the PDR (black curve) and the NDR (gray curve), $\Omega=2 \pi \times 50 \mathrm{kHz}, \Theta=2 \pi \times 5 \mathrm{kHz}$.
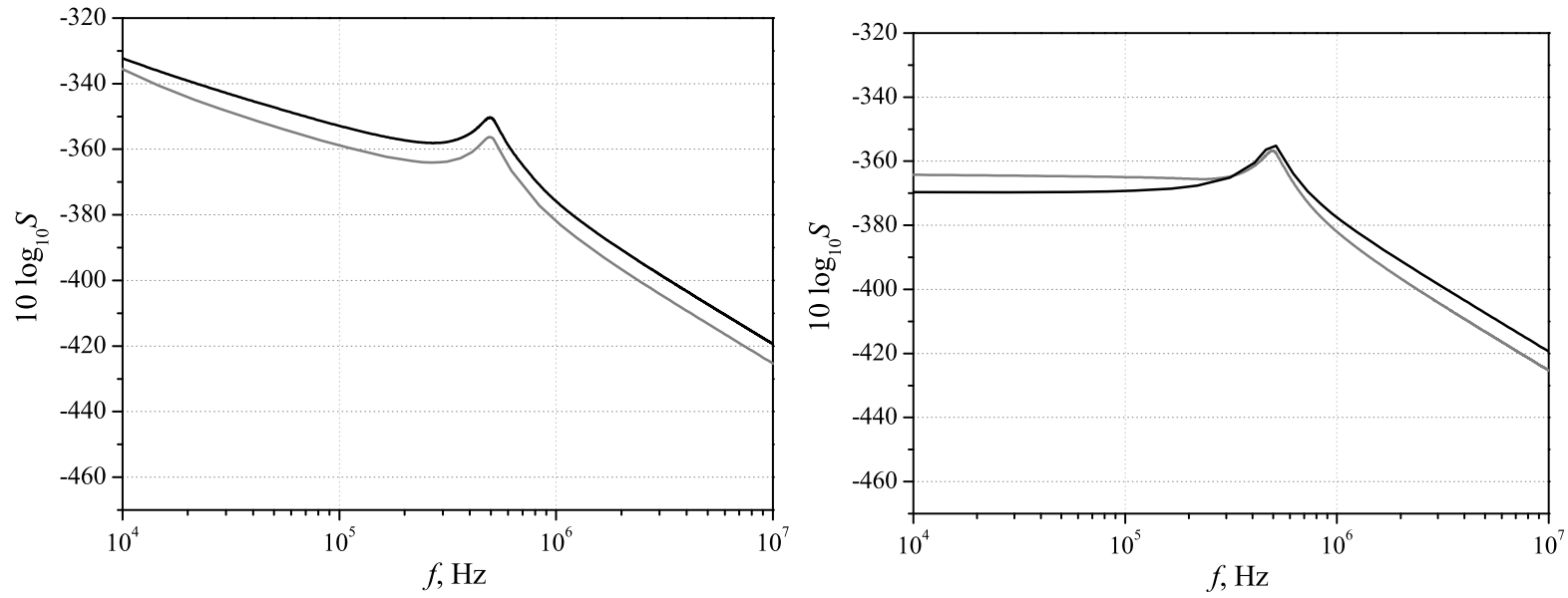

Figure 11. Noise spectrum induced by gain fluctuation in the NDR (left) and the PDR (right). $\Omega_{g}=5.3 \mathrm{THz}$ (black curves) and 10.5 THz (gray curves). Other parameters correspond to Fig. 10.

and the net-noise spectra in the NDR (gray curve) and the PDR (black curve) are shown in Fig. 10 (right). From comparison of the left and right Figs. 10, one can see that the noise exceeds the quantum limit in the vicinity of the resonant peak, which corresponds to the frequency of the gain modulation. The low-frequency branch demonstrates the noise-excess in the NDR (gray curve, right Fig. 10) in comparison with the PDR (black curve, right Fig. 10). Simultaneously, the noise in the PDR with oscillating gain exceeds the quantum limit substantially.

The gainband broadening, which damps the gain dispersion, reduces the noise in the NDR (left Fig. 11. compare the gray and black curves as well as see left Fig. 6). In the PDR, the gainband broadening enhances the low-frequency noise but reduces the high-frequency noise (the right Fig. 11] compare the gray and black curves as well as see the left Fig. (6).

\section{CONCLUSIONS}

The numerical analysis of a mode-locked Yb:YAG thin-disk oscillator operating in both PDR and NDR is presented. The energy scalability within a broad energy range (from 6 to $100 \mu \mathrm{J}$ ) is analyzed. It is found, that the level of stabilizing GDD is substantially reduced in the PDR $\left(\approx 0.003-0.01 \mathrm{ps}^{2}\right.$ vs. $-0.02--0.3 \mathrm{ps}^{2}$ in the NDR). The pulse duration in the PDR is approximately tenfold of that in the NDR, but the spectra in the PDR 
is broader. These spectra is truncated, asymmetrical and concave. In spite of the pulse duration growth, the spectra broaden with energy for a chirped pulse.

The timing jitter of a mode-locked Yb:YAG thin-disk oscillator is analyzed numerically. As a source of jitter, the gain fluctuations are considered. It is found, that the timing jitter is substantially reduced in the PDR. It is assumed, that the mechanism of such reduction is a negative passive feedback induced by spectral dissipation. We see, that, in general, the difference between the levels of timing jitter in the PDR and the NDR decreases with a gainband broadening, i.e. with transit from a solid-state to a fiber oscillator. Scaling properties of timing jitter depend on the regime: the jitter increases with energy for the PDR and decreases for the NDR. The dependence on dispersion differs, as well: in spite of the NDR, the timing jitter grows with dispersion in the PDR. The temporal and spectral coherence is reduced due to jitter for both regimes, but the reduction is lower for a chirped pulse.

Our simple analytical analysis demonstrates that the quantum timing noise is substantially reduced in the PDR due to suppression of the frequency fluctuations. The difference between the noise levels of the PDR and the NDR decreases with frequency because the timing noise due to spontaneous emission is higher for the PDR as a result of larger pulse duration. The analysis confirms the numerical result that the timing jitter induced by gain fluctuations is suppressed in the PDR in comparison with the NDR for low-frequencies of the noise. The gainband broadening reduces this noise in the NDR and in the PDR (for high-frequencies). Simultaneously, the low-frequency noise induced by gain fluctuations in the PDR increases with the gain broadening.

\section{ACKNOWLEDGMENTS}

The work was supported by Austrian Fonds zur Förderung der wissenschaftlichen Forschung (project P20293) and Munich Centre for Advanced Photonics (MAP).

\section{REFERENCES}

[1] T.Südmeyer, S.V.Marchese, C.R.E.Baer, G.Gingras, B.Witzel, U.Keller, "Femtosecond laser oscillators for high-field science," Nat. Photonics 2, pp.599-604, 2008.

[2] T.Südmeyer, C.Kränkel, C.R.E.Baer, O.H.Heckl, C.J.Saraceno, M.Golling, R.Peters, K.Petermann, G.Huber, U.Keller, "High-power ultrafast thin disk laser oscillators and their potential for su-100femtosecond pulse generation," Appl. Phys. B 97, pp.281-295, 2009.

[3] S.V.Marchese, C.R.E.Baer, A.G.Engqvist, S.Hashimoto, D.J.H.C.Maas, M.Golling, T.Südmeyer, U.Keller, "Femtosecond thin disk laser oscillator with pulse energy beyond the 10-microjoule level," Opt. Express 16, pp.6397-6407, 2008.

[4] J.Neuhaus, D.Bauer, J.Zhang, A.Killi, J.Kleinbauer, M.Kumkar, S.Weiler, M.Guina, D.H.Sutter, Th.Dekorsy, "Subpicosecond thin-disk laser oscillator with pulse energies of up to 25.9 microjoules by use of an active multipass geometry," Opt. Express 16, pp.20530-20539, 2008.

[5] G.Palmer, M.Schultze, M.Siegel, M.Emons, U.Bünting, U.Morgner, "Passively mode-locked $\mathrm{Yb}: \mathrm{KLu}\left(\mathrm{WO}_{4}\right)_{2}$ thin-disk oscillator operated in the positive and negative dispersion regime," Opt. Lett. 33, pp.1608-1610, 2008.

[6] Ch.Gohle, Th.Udem, M.Herrmann, J.Rauschenberger, R.Holzwarth, H.A.Schuessler, F.Krausz, Th.W.Hänsch, "A frequency comb in the extreme ultraviolet," Nature 436, pp. 234-237, 2005.

[7] Y.Liu, S.Tschuch, A.Rudenko, M.Durr, M.Siegel, U.Morgner, R.Moshammer, J.Ullrich, "Strong-field double ionization of Ar below the recollision threshold," Phys. Rev. Lett. 101, pp.053001-1-053001-4, 2008.

[8] G.A.Mourou, T.Tajima, S.V.Bulanov, "Optics in the relativistic regime," Rev. Mod. Phys. 78, pp.309-371, 2006.

[9] S.Namiki, E.P.Ippen, H.A.Haus, Ch.X.Yu, "Energy rate equations for mode-locked lasers," J. Opt. Soc. Am. B 14, pp.2099-2111, 1997.

[10] V.L.Kalashnikov, A.Apolonski, "Chirped-pulse oscillators: a unified standpoint," Phys. Rev. A 79, pp.043829-1-043829-10, 2009. 
[11] J.Neuhaus, D.Bauer, J.Kleinbauer, A.Killi, D.H.Sutter, Th.Dekorsy, "Numerical analysis of a subpicosecond thin-disk laser oscillator with active multipass geometry showing a variation of pulse duration within one round trip," J. Opt. Soc. Am. B 27, pp.65-71, 2010.

[12] V.L.Kalashnikov, "The unified theory of chirped-pulse oscillators," Proc. SPIE 7354, pp.73540T-1-73540T$12,2009$.

[13] H.A.Haus, A.Mecozzi, "Noise of mode-locked lasers," IEEE J. Quantum Electron. 29, pp.983-996, 1993.

[14] R.Paschotta, "Noise of mode-locked lasers (Part II): timing jitter and other fluctuations," Appl. Phys. B 79, pp.163-173, 2004.

[15] R.Paschotta, "Noise of mode-locked lasers (Part I): numerical model," Appl. Phys. B 79, pp.153-162, 2004.

[16] R.Paschotta, "Timing jitter and phase noise of mode-locked fiber lasers," Optics Express 18, pp.5041-5054, 2010.

[17] K.E.Oughstun, Electromagmetic and Optical Pulse Propagation 1, Springer, NY, 2006.

[18] C.Paré, P.-A.Bélanger, "Optical solitary waves in the presence of a Lorentzian gain line: limitations of the Ginzburg-Landau model," Optics Commun. 145, pp.385-392, 1998.

[19] Ch.-W. Chang, S.Chi, "Ultrashort pulse generation from mode-locked erbium-doped fibre ring lasers," $J$. Modern Optics 46, pp.1431-1442, 1999.

[20] F.X.Kärtner (Ed.), Few-cycle Laser Pulse Generation and its Applications, Berlin, Springer Verlag, 2004.

[21] N.N.Akhmediev, A.Ankiewicz, (Eds.), Dissipative Solitons, Berlin, Springer Verlag, 2005.

[22] V.L.Kalashnikov, "Chirped dissipative solitons," arXiv:1001.4918.

[23] S.Uemura, K.Torizuka, "Profile analysis of a Yb:YAG chirped-pulse oscillator ," in Advanced Solid-State Photonics Technical Digest, San Diego, p.AWB25, 2010.

[24] V.L.Kalashnikov, E.Podivilov, A.Chernykh, S.Naumov, A.Fernandez, R.Graf, A.Apolonski, "Approaching the microjoule frontier with femtosecond laser oscillators: theory and comparison with experiment," New J. Physics 7, pp. 217-1-217-16, 2005.

[25] V.L.Kalashnikov, "Chirped dissipative solitons of the complex cubic-quintic nonlinear Ginzburg-Landau equation," Phys. Rev. E 80, pp.046606-1-046606-8, 2009.

[26] I.L.Budunoğlu, C.Ülgüdür, B.Oktem, F.Ö.Ilday, "Intensity noise of mode-locked fiber lasers," Optics Lett. 34, pp.2516-2518, 2009.

[27] T.R.Schibli, I.Hartl, D.C.Yost, M.J.Martin, A.Marcinkevičius, M.E.Fermann,J.Ye, "Optical frequency comb with submillihertz linewidth and more than $10 \mathrm{~W}$ average power," Nat. Photonics 2, pp. 355-359, 2008.

[28] J.M.Dudley, S.Coen, "Coherence properties of supercontinuum spectra generated in photonic crystal and tapered optical fibers," Optics Lett. 27, pp. 1180-1182, 2002.

[29] C.Y.Teisset, H.Fattahi, A.Sugita, L.Turi, X.Gu, O.Pronin, V.Pervak, F.Kraus, A.Apolonski, "700 nJ broadband MHz optical parametric amplifier," in Ultra Fast Optics and High Field Short Wavelength Conference Program, Arcachon, 2009. 